\title{
A Complex Quantity Slide Rule
}

\author{
BY JESSE W. M. DuMOND ${ }^{2}$
}

Synopsis. - The need for a device to shorten numerical work with complex quantities is pointed out and a description is

given of a slide rule in two dimensions devised to fill this need.

$\mathrm{T}$ HE principle of the ordinary slide rule can be extended into two dimensional space and applied to complex quantities.

Up to the present time, complex quantities have been employed almost exclusively for theoretical analysis and relatively little in numerical work. Formulas developed by means of complex quantities are generally split into components or otherwise transformed so that the numerical work is always performed with ordinary numbers. Unfortunately in many cases, formulas indeed very simple, when expressed as functions of complex quantities, have very much more elaborate expressions when thus transformed to adapt them to ordinary numerical work. An example of this is the propagation constant of a transmission line.

$$
n=\sqrt{(R+j X)(G+j B)}
$$

which becomes upon transformation

$$
\begin{aligned}
\frac{1}{\sqrt{ } 2} & {\left[\sqrt{\left(R^{2}+X^{2}\right)\left(G^{2}+B^{2}\right)}+(R G-X B)\right]^{\frac{1}{2}} } \\
& +j \frac{1}{\sqrt{2}}\left[\sqrt{\left(R^{2}+X^{2}\right)\left(G^{2}+B^{2}\right)}-(R G-X B)\right]^{\frac{1}{2}}
\end{aligned}
$$

Here the advantage of treating the complex quantities as numerical entities and evaluating merely the formula

$$
n=\sqrt{Z Y}
$$

is evident. Seventeen numerical operations would thus be reduced to two, namely, one multiplication and one extraction of the square root.

To date, the use of complex quantities in arithmetical work has been hampered by the two following obstacles, both of which are removed by the complex quantity slide rule.

1. Frequently the known quantities are the phaseangle and modulus (effective value of current or voltage) whereas it may be necessary for calculation to transform this into a complex quantity in the component form. This requires a trigonometric transformation from modulus-phase-angle form to component form and frequently a second transformation of the final result from component form back to modulus-phase-angle form.

2. Ordinary numerical operations upon complex quantities are long. Multiplication and division are

1. This paper is an elaboration and an improvement of some ideas originally offered by the author as a thesis for the Master's Degree at Union College, Schenectady, New York.

2. Teaching Fellow, California Institute of Technology.

Presented at the Pacific Coast Convention of the A.I.E.E., Pasadena, Cal., October 13-17, 1924. performed at present either by elementary algebraic methods, including multiplication of the conjugate of the divisor in division or by the modulus-argument method recommended by Kennelly, which necessitates trigonometric transformations. By the first method each multiplication requires four numerical operations of multiplication and two operations of addition or subtraction, a total. of six operations. Each division requires two operations of squaring, three operations of addition or subtraction, four operations of multiplication and two operations of division, a total of eleven operations. By the second method each transformation from component form to modulus-argument form requires two divisions and looking in tables for the anti-tangent and the sine or cosine of one angle. Each transformation in the reverse direction requires looking in tables for the sine and cosine of one angle and two multiplications. When these transformations are made, two more operations one of multiplication or division, the other of addition or subtraction are necessary to effect a division or multiplication of the complex quantities. This is only slightly shortened by the use of ordinary logarithms.

It is very probable that complex quantities will become more and more useful to engineers and scientists as time goes on. It is also very likely that the most prominent obstacle to a wider application of them at present is precisely the above mentioned cumbersomeness which they possess in numerical work. With such an obstacle removed, many as yet undeveloped applications for them, would soon appear. In the domain of electricity alone, alternating current power transmission, telephony and radio, call for many applications of complex quantities. Their utility extends much farther offering as they do, very powerful methods of mathematical analysis applicable to a great variety of physical problems.

\section{The Complex Quantity Slide Rule}

It is possible to extend the principle of the ordinary or Mannheim slide rule to include complex quantities. The principle remains that of the graphical addition of logarithms, but the two degrees of freedom possessed by complex quantities, necessitates that the slide rule be extended over a plane surface.

In the ordinary engineer's slide rule the distance measured in appropriate units from the left extremity of the scale to any division on the rule is the logarithm of the number associated with that division.

In the complex quantity slide rule, the position of a point in a plane defined with reference to two rec- 
tangular coordinate axes represents a complex quantity. This complex quantity is the logarithm of the complex quantity associated with the point.

Just as points on the ordinary slide rule are located by means of a set of non-uniform linear divisions, so on the complex quantity slide rule points are located by means of a system of curvilinear coordinates. These curvilinear coordinates consist of two mutually intersecting families of curves, a "real" family and an "imaginary" family, each curve having an associated number, the numbers ranging from one to ten. The curves are so arranged that the point at the intersection of any pair of curves referred to the rectangular reference axes graphically represents the logarithm of the complex quantity associated with that pair of curves.

\section{The Curvilinear Coordinate System}

Fig. 1 represents the system of curvilinear coordinates. The reference axes are shown by the heavy lines but since these are not necessary for the operation of the slide rule, they do not ordinarily appear upon it. The point $P$ indicated in the figure is at the intersection of the curve +3 of the real system and +4 of the imaginary system and its coordinate distances from

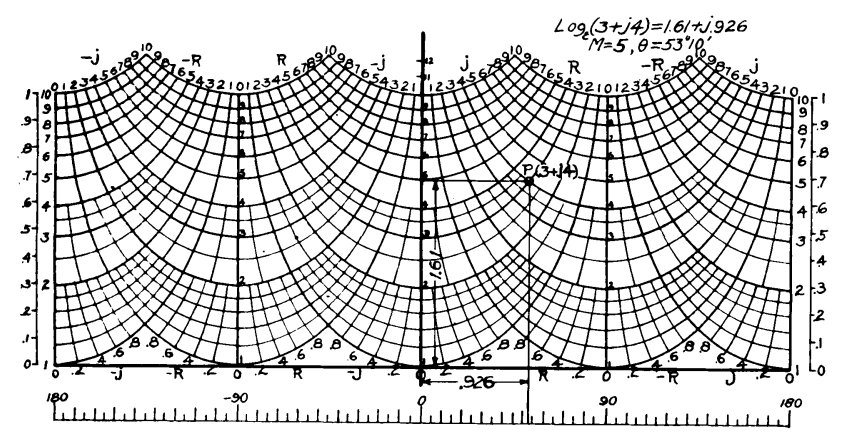

FIG. 1

the reference axes measured in appropriate units are 1.61 and 0.926 respectively. The Naperian complex logarithm of $(3+j 4)$ is $(1.61+j 0.926)$.

It will be noted that the system is divided into four similar patterns and that the numbers of the curves intersecting in each pattern differ only in algebraic sign. These signs are $(-,-)(+,-)(+,+)$ and $(-,+)$ naming the four patterns consecutively from left to right and giving in each case the sign of the "real" curve first. It is evident then that all complex quantities, whatever signs be associated with the real and imaginary components, have corresponding points on the system and each of the four patterns includes only those points whose corresponding complex quantities fall in a given quadrant in the ordinary clock or Argand diagram. In fact the horizontal distance measured in suitable units from the central vertical reference axis to any point in the system is equal to the argument or phase angle of the complex quantity associated with that point. Moreover the vertical distance measured again in suitable units up from the horizontal reference axes to any point in the system is the logarithm of the modulus of the complex quantity associated with that point. The reasons for these facts will be found in the appendix. Given a point on the system of curvilinear coordinates it is thus possible to read from the curves the "real" and "imaginary" components of the complex quantity associated with that point or to read the phase angle and modulus of the complex quantity by aligning the point with the horizontal scale of angles and with the vertical scale of moduli formed by the intersections of the curves with any one of the five vertical straight lines. The alignment is accomplished by a mechanical means to be described below.

\section{The Curies as a Distorted System}

The curvilinear coordinates may be derived by the proper distortion of a set of ordinary rectangular coordinates. This distortion will be described because it will serve the purpose of giving the shape of the curves and the operations they perform a clearer meaning.

If we subject a system of ordinary rectangular coordinates to a process of expansion and contraction such that all differential linear magnitudes at any point a distance $r$ from the origin will change in the ratio $\frac{1}{r}$ then all parts of the system outside a unit circle about the origin will shrink and all points inside the unit circle will expand until the final shape assumed will be a cylinder of unit radius. The system of curvilinear coordinates shown is what would be obtained by developing this cylinder upon a plane.

Any straight line drawn on the original system of rectangular coordinates through the origin (this includes the two principle axes) will swing about an axis tangent to the unit circle where the line in question cuts the unit circle, very much as the ribs of an umbrella swing about the small circle to which they are attached while the umbrella is being closed. Meanwhile, the length elements of these lines will have shrunk for those parts originally outside the unit circle so that what were equal divisions will now have become much more crowded as the distance from the unit circle increases. Fig. 2 shows the initial and final states in this distortion process.

It will be clear from Fig. 2 that the principle axes of the original cartesian coordinates become the five vertical straight lines of Fig. 1, the end lines being coincident on the cylinder. It will also be clear why horizontal distances in Fig. 1 represent phase angles of complex quantities in the original system. Circles about the origin of the original cartesian coordinates as center become horizontal straight lines after distortion.

The horizontal separation of any two points on the rule measures the phase angle between the corresponding complex quantities. 


\section{DesCRIPTION OF RUle IN Simplest Form}

In order to utilize the above described system of curves it is necessary to provide a means of graphically adding the complex logarithms which they serve to locate. This is accomplished by an element bearing these curves which is free to translate in any direction in a plane. This element shown at $A$, Fig. 3 , occupies an initial position near the center of the board $B$. It can be repeatedly returned to this position by bring-

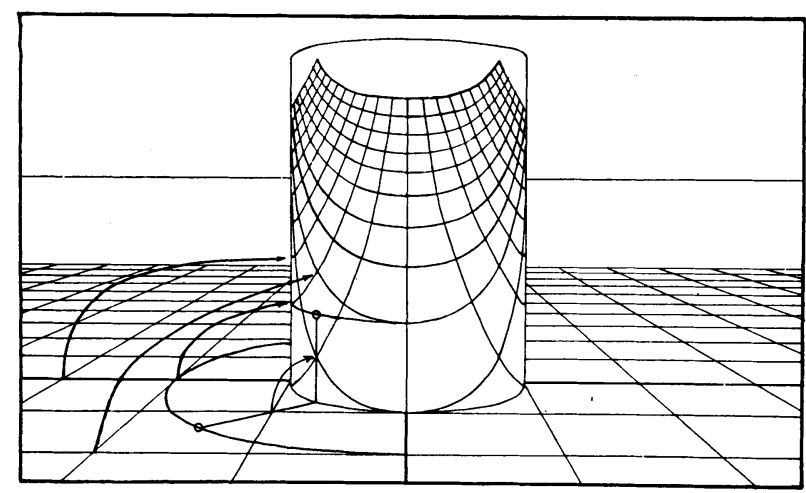

FIG. 2

ing certain fiducial marks $f$ on the board and on the transparent insert of element $A$ into coincidence. A second element $C$ consisting of a jointed transparent celluloid arm bears a small black point which can be made to coincide with any point on the curvilinear chart $A$ and will maintain the position of that point relative to the board $B$ unchanged while the element $A$ is undergoing translations.

The ordinary slide rule differs from the complex

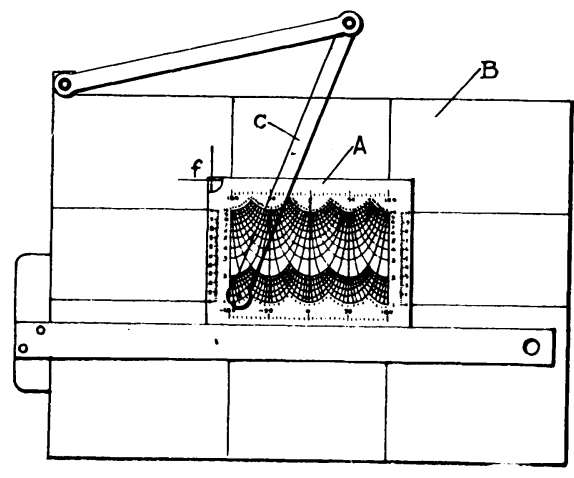

FIg. 3

quantity slide rule in that the former is provided with both a stationary and a moveable scale while the latter has but one system of curvilinear coordinates. The operation of multiplication is performed as follows: With $A$ in its initial position the pointer $C$ is set to coincide with one of the complex quantities to be multiplied. The element $A$ is then translated so as to bring $(1+j 0)$ or $(10+j 0)$ under the pointer $C$. The pointer $C$ is then set to coincide with the second complex quantity to be multiplied. The product is read off by returning the element $A$ to its initial position and reading the curves which now intersect under the pointer. The evaluation of a fraction containing factors in numerator and denominator can be rapidly accomplished by a similar process.

Some care must be exercised as regards the decimal point in setting complex quantities on the rule. The curves are numbered up to 10 and in order to accomodate complex quantities whose components exceed 10 the decimal point is shifted the necessary amount in both components of the complex quantity. This shift is to be chosen so as to make the larger component fall between one and ten. It is important that both components should undergo the same decimal point shift.

The same difficulty of "running off scale" which occurs in the use of the ordinary slide rule appears in the complex quantity slide rule. This difficulty is avoided in a similar way and is completely absent in the improved form of the rule. It is evident that a displacement of either the rule or the pointer through a vertical distance equal to the distance on the rule from

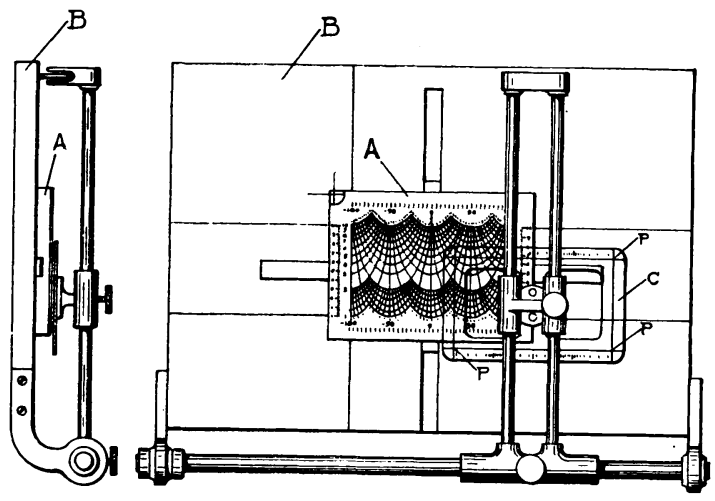

Fig. 4

$(1+j 0)$ to $(10+j 0)$ affects only the decimal point of the result. A horizontal displacement through a distance equal to the horizontal length of the rule has no effect on the result since it is equivalent to rotating the complex quantity through a phase angle of 360 . If then a point falls off scale it suffices to move either the rule or the pointer through one or both of the above displacements to bring it back on scale.

\section{IMPROVED FORM OF RULE}

Fig. 4 shows an improved form of the complex quantity slide rule.

The translatory movement of the chart $A$ is guided by a cross arm device sliding in grooves in the top of the board $B$ and the bottom of the element $A$.

The pointer is replaced by a celluloid rectangle $C$ bearing two pairs of intersecting straight lines forming a rectangle whose length is equivalent to a displacement of $360 \mathrm{deg}$. and whose height is equivalent to a displacement from $(1+j 0)$ to $(10+j 0)$.

Any of the intersections or corners $P$ of this rectangle may be used interchangeably as the fiducial point in 
operations upon complex quantities since an interchange of corners only affects the position of the decimal point in the result. Since one of these corners must always be on the curvilinear chart there can be no difficulty from running off scale.

The straight lines on the "indicator" as the celluloid rectangle is called serve the purpose of aligning the point in question with a scale of phase angles provided on the top and bottom edges of the rule and also with the scales of moduli formed by the intersections of the curves with any one of the vertical straight lines. There are vertical scales of equal parts on either end of the rule which permit by means of these same lines on the indicator to read off the logarithms of the modulus of a complex quantity. In Fig. 4 the indicator is shown set on $(3+j 4), M=5, \theta=53 \mathrm{deg} .10 \mathrm{~min}$.

The indicator is also provided with horizontal cosine or power factor scales which permit of reading directly
It is thus possible by means of the complex quantity slide rule to perform multiplication, division, involution and evolution of numerical complex quantities as well as to find the logarithms of complex quantities. The time saved over what is required by ordinary longhand methods is very great. A single example will suffice to illustrate this.

\section{IlluSTRATIVE EXAMPLE}

The current that a certain generator must supply when a transmission line is short circuited at the receiving end is given by

$I=81200 \frac{(0.920+j 0.0356)(0.000445+j 0.002062)}{(0.0815+j 0.402)(0.32+j 0.77)}$

Shifting the decimal points as explained above by equal amounts in one and the same complex quantity so as to bring the larger component in each case to a value between unity and ten gives

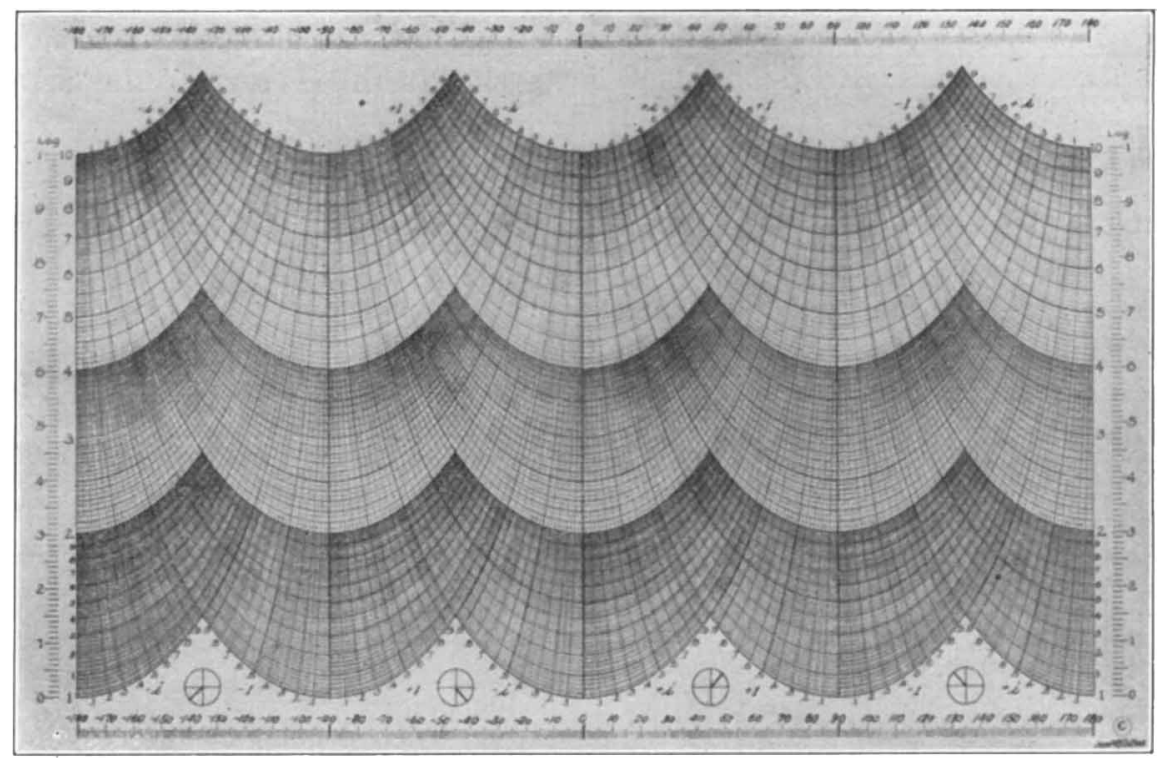

Fig. 5-Element "A" of Slide Rule

the cosine of the angle between a point on the rule representing a current and a second point representing a voltage.

The horizontal and vertical scales of equal parts on the rule permit of reading directly the phase angle and the logarithms of the modulus of any complex quantity. A second complex quantity formed of these two readings with the angle as the imaginary component is the common logarithm of the original complex quantity. (In order to obtain the natural or Naperian complex logarithm the units would have to be reduced to radians and base $\epsilon$ logarithms.) If then any power or root of a complex quantity is desired it is sufficient to multiply or divide the readings of these two scales by the index of the power or root. The power or root is then read off the curvilinear chart at the point which is in alignment with the new dividend or multiplied readings on the scales.

$$
I=\frac{(9.20+j 0.356)(0.445+j 2.062)}{(0.815+j 4.02)(3.2+j 7.7)} \times 8.12 \times 10^{2}
$$

Set the rule in the initial position. Set the indicator over the first complex quantity $(9.20+j 0.356)$. Move the rule so as to bring the indicator. over the quantity $(0.815+j 4.02)$. Move the indicator over $(0.445+j 2.062)$. Move the rule so as to bring the indicator over $(3.2+j 7.7)$. Finally move the indicator over $(8.12+j 0)$. Return the rule to its initial position. The result found under the indicator is $(1.87-j 4.22)$.

The current is then

$$
I=(1.87-j 4.22) 10^{2}=187-j 422
$$

The modulus or magnitude of the short-circuit current is read off where the horizontal line of the indicator crosses any one of the vertical lines of the rule. It is $M=4.61 \times 10^{2}=461$ amperes. Without 
disturbing the indicator the phase angle may be read off. It equals $-66 \mathrm{deg}$.

In this problem the voltage was assumed to be on the axis of reals. The power factor is read off the cosine scale on the indicator as 0.41 and it is lagging since the phase angle of the current is negative.

\section{Takes Care of Algebraic Signs}

The work here accomplished with five settings of the rule would require some twenty operations with tables and logarithms or with an ordinary slide rule. Moreover the danger of mistakes in algebraic signs is largely eliminated because these are automatically cared for on the complex quantity slide rule if the settings are made correctly. The complex quantity slide rule goes definitely beyond the ordinary slide rule in this point of its ability to handle algebraic signs. Unlike the ordinary slide rule the value zero for either real or imaginary components appears on the rule. The complex quantity $(0+j 0)$ does not appear however.

\section{Transcendental FunCtions}

In many cases transcendental functions of complex quantities such as the hyperbolic and trigonometric

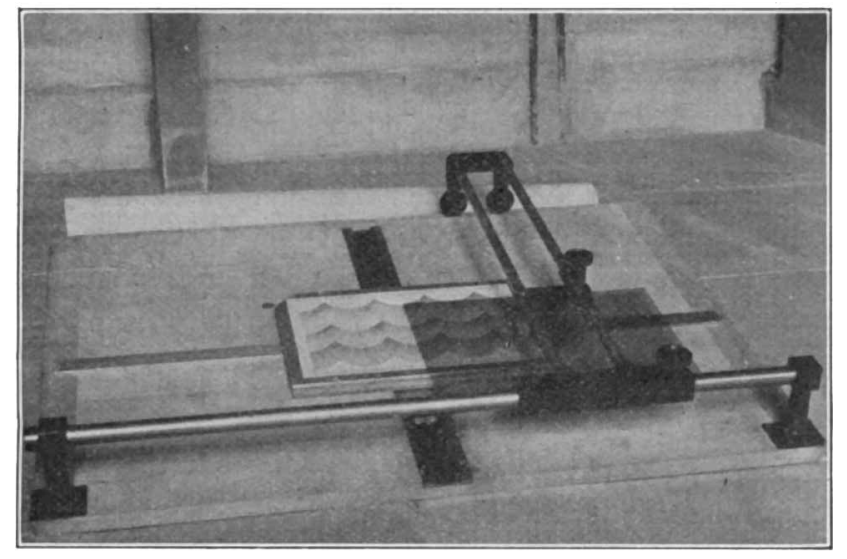

Fig. 6-Complex Quantity Slide Rulf

functions are called for. Tables for such functions of a complex variable exist so that quantities taken from such tables could be employed directly on the rule. It is also possible to construct a complex quantity slide rule with interchangeable elements bearing curvilinear systems of coordinates adapted to perform operations with the transcendental functions. One difficulty, however, presents itself here. The network of curves shown in Fig. 1 if extended in all directions over an infinite plane so as to include all possible values of the complex variable, would be made up merely of an infinite number of repetitions of the pattern element as shown in Fig. 5, extending above, below and on all sides of it. It is this quality of multiplicity which makes it possible to operate with merely one element of the pattern, for homologous points in the different elements differ in absolute magnitude merely by multiples of ten and in phase angle merely by multiples of
360 deg. But in the case of a set of curvilinear coordinates adapted to some transcendental function of the complex variable, this convenient repetitive property no longer obtains. This places a rather awkward limitation on the utility of such special functional coordinates.

Fortunately, in the case of the hyperbolic functions, the complex quantity slide rule affords another method without modification of the curvilinear coordinates. This is because the hyperbolic functions of a complex variable can be simply expressed as exponentials or antilogarithms. These antilogarithms can be taken from the complex quantity slide rule by a process the reverse of that described for finding logarithms. Thus $\sinh (a+j b)$, cosh $(a+j b)$ and $\tanh (a+j b)$ can be found with the complex quantity slide rule without reference to tables. A detailed description of these special applications goes somewhat beyond the purpose of the present paper which is merely to introduce the device to the engineering fraternity. It is evident, however, that many formulas in use today in connection with alternating current work can be considerably simplified by modifications which would adapt them for use with the complex quantity slide rule. The ease with which ordinary analytic operations on complex quantities are performed with this device makes it feasible to compute infinite series and infinite products of a complex variable and thus to obtain numerical values of definite integrals and other functions heretofore practically prohibited by the labor of computation.

It is not to be supposed that the complex quantity slide rule is a pons asinorum to eliminate thinking. It requires perhaps even a clearer comprehension of complex quantities on the part of the operator than would be required by the older methods. It is a substitute for drudgery, not for intelligence.

\section{Appendix}

The logarithm of a complex quantity is itself another complex quantity. To find the components $\alpha$ and $\beta$ of $\log (a+j b)$ let

$$
\begin{array}{cc} 
& \log _{\epsilon}(a+j b)=\alpha+j b \\
\text { then } & \epsilon^{\alpha+j \beta}=a+j b \\
& \epsilon^{\alpha}(\cos \beta+j \sin \beta)=a+j b
\end{array}
$$

Equating reals and imaginaries separately gives

$$
\begin{aligned}
& \epsilon^{\alpha} \cos \beta=a \\
& \epsilon^{\alpha} \sin \beta=b
\end{aligned}
$$

Squaring and adding

$$
\begin{gathered}
\epsilon^{2^{\alpha}}=a^{2}+b^{2} \\
\quad \alpha=\log _{\epsilon} \sqrt{a^{2}+b^{2}}
\end{gathered}
$$

Dividing one by the other gives

$$
\begin{aligned}
\tan \beta & =\frac{b}{a} \\
\beta & =\tan ^{-1} \frac{b}{a}
\end{aligned}
$$


so that we can write

$\log _{\epsilon}(a+j b)=\log _{\epsilon} \sqrt{a^{2}+b^{\llcorner}}+j \tan ^{-1} \frac{b}{a}$

The lower extremity of the central vertical line on the complex quantity slide rule is the origin point. The position of any point on the rule referred to vertical and horizontal cartesian coordinates through this origin point graphically represents the logarithm of the complex quantity associated with that point. The real curves are the loci of all points whose associated complex quantities have one and the same real value (and a variable imaginary value) and this value is the number printed beside the curve. The imaginary curves are the loci of all points whose associated complex quantities have one and the same imaginary value.

To find the equations expressing the loci, consider any point on the rule with which the complex quantity $(a+j b)$ is associated.

The coordinates of this point are

$$
\begin{aligned}
& y=\log \sqrt{a^{2}+b^{2}} \\
& x=\tan ^{-1} \frac{b}{a}
\end{aligned}
$$

(The interchange of coordinates, $y$ being taken for the axis of reals here is purely for convenience). The real loci are obtained by eliminating $b$ between these two equations and the imaginary loci are obtained by eliminating $a$. This gives

$$
\begin{aligned}
y & =\log a-\log \cos x \\
y^{1} & =\log b-\log \sin x
\end{aligned}
$$

as the equations of the real and imaginary systems of curvilinear coordinates. The system is seen to be a conformal transformation of the type $W=\log y$. If the curves are plotted to scales of equal units for both $x$ and $y$, all the intersections will be rectangular. This is not done in the actual rule as it yields a more convenient shape to compress the $x$-coordinates somewhat. It will also be noted that the curves consist of a characteristic part $(-\log \cos x)$ and an additive constant, $(\log a)$. In other words, all the curves on the rule are intrinsically identical and differ merely in their position.

From equations (5) and (6) it evident that at any point on the rule corresponding to $(a+j b)$ the $y$ coordinate is the logarithm of the absolute magnitude of $(a+j b)$ and the $x$-coordinate is the phase angle of $(a+j b)$. This explains the horizontal scale of phase angles. The method of reading the absolute magnitudes directly from the vertical scale formed by the intersections of the curves with any one of the vertical straight lines will be understood from the following.

Consider the equations (7) and (8) for the double system of curves already given. From (7) it appears that when, $a=0$, the equation of the "real" curve becomes

$$
x= \pm \frac{\pi}{2}
$$

and from (8) when, $b=0$, the equation of the "imaginary" curve becomes

$$
x=0, \pm \pi
$$

In other words at these points respectively the real and imaginary systems have straight vertical loci. In each case the intersections of these straight vertical loci with the other system of curves may be found by substituting the values (10) into (7) and the values (9) into (8), giving

$$
\begin{aligned}
& y=\log a \\
& y^{\prime}-=-\log -b
\end{aligned}
$$

Thus it is seen that the $y$-coordinate of the point of inter-section of each of the curves with the vertical straight lines is in each case the logarithm of the number associated with that curve. Thus

$$
y=\log a
$$

The $y$-coordinate of any point on the rule is the logarithm of the absolute magnitude of the complex quantity associated with that point.

$$
y=\log M
$$

Hence, if the point is horizontally aligned with some point on a vertical straight line we have

$$
\begin{gathered}
\log a=y=\log M \\
a=M
\end{gathered}
$$

so that by reading the value of $a$, on any vertical straight line at the same vertical height as the point in question the absolute magnitude of the complex quantity associated with that point is found.

\section{Curvilinear System as a Distorted Cartesian SYSTEM}

Consider first the cartesian system, but divide it into infinitesmal elements by a system of radial straight lines and circles concentric about the origin. If we subject this to the distortion

$$
d s^{\prime}=\frac{d s}{r}
$$

where $r$ is the radial distance from the origin to the arbitrarily oriented linear element, $d s$, then it will be self evident that all of the concentric circles will assume unit radius, all of the elements of area previously included between adjacent radii and adjacent circles will become rectangles on a cylindrical surface and the radial lines will become elements of a cylinder of unit radius.

Consider a point which before distortion was at a radial distance, $r$, from the origin. All elements of this radius will shrink to $d r^{\prime}=\frac{d r}{r}$. After distortion this point will be at a distance $r^{\prime}$ from the original unit circle on the cylinder.

$$
r^{\prime}=\int_{r=1}^{r=r} d r^{\prime}=\int_{r=1}^{=r} \frac{d r}{r}=\log r
$$


The distortion may be treated as follows.

The equations of the curves in the cartesian coordinates were

Now

$$
y=b, \quad x=a
$$

$$
d s=\sqrt{d y^{2}+d x^{2}}
$$

and

$$
\begin{aligned}
& d s^{\prime}=\frac{d s}{r}=\frac{\sqrt{d y^{2}+d x^{2}}}{\sqrt{x^{2}+y^{2}}}=\sqrt{d x^{\prime 2}+d y^{\prime 2}} \\
& d x^{\prime 2}+d y^{\prime 2}=\frac{d x^{2}+d y^{2}}{x^{2}+y^{2}}
\end{aligned}
$$

This is the only condition imposed by the distortion. We may choose the value of either $d x^{\prime}$ or $d y^{\prime}$ in any arbitrary way compatible with the distortion. Thus we may say

$d y^{\prime}=\frac{d r}{r}=\frac{d \sqrt{x^{2}+y^{2}}}{\sqrt{x^{2}+y^{2}}}=\frac{x d x+y d y}{x^{2}+y^{2}}$

Then

$$
\begin{aligned}
& d y^{\prime 2}=\frac{(x d x+y d y)^{2}}{\left(x^{2}+y^{2}\right)^{2}} \\
& d x^{\prime 2}=\frac{d x^{2}+d y^{2}}{x^{2}+y^{2}}-\frac{(x d x+y d y)^{2}}{\left(x^{2}+y^{2}\right)^{2}} \\
& d x^{\prime 2}=\frac{x^{2} d y^{2}-2 x y d x d y+y^{2} d x^{2}}{\left(x^{2}+y^{2}\right)^{2}}
\end{aligned}
$$

$$
=\left(\frac{x d y-y d x}{x^{2}+y^{2}}\right)^{2}
$$

$d x^{\prime}=\frac{x d y-y d x}{x^{2}+y^{2}}$

Integrating

$y^{\prime}=\int \frac{x d x+y d y}{x^{2}+y^{2}}+c, x^{\prime}=\int \frac{x d y-y d x}{x^{2}+y^{2}}+c_{1}$

for the curve

$$
\begin{gathered}
x=a, \quad d x=0 \\
y^{\prime}=\int \frac{y d y}{a^{2}+y^{2}}+c, \quad x^{\prime}=\int \frac{a d y}{a^{2}+y^{2}}+c_{1} \\
y^{\prime}=\log \sqrt{a^{2}+y^{2}}+c, x^{\prime}=\tan ^{-1} \frac{y}{a}+c_{1}, \\
\text { or } y=\log a-\log \cos x^{\prime}
\end{gathered}
$$

similarly for the curve

$$
\begin{gathered}
y=b, \quad d y=0 \\
y^{\prime}=\log \sqrt{b^{2}+x^{2}}+c, x^{\prime}=\tan ^{-1} \frac{b}{x}+c_{1}, \\
\quad \text { or } y^{\prime}=\log b-\log \sin x^{\prime}
\end{gathered}
$$

if we choose $c$ and $c_{1}$ arbitrarily equal to zero which amounts to fixing the origin point.

\section{ARTIFICIAL ILLUMINATION OF POUL- TRY HOUSES FOR WINTER EGG PRODUCTION}

The fact that the use of artificial light has been found in some instances, to stimulate winter egg production by as much as 70 per cent, has brought up the question as to what intensity is conducive to maximum production. The answer to this was obtained only after an extended investigation covering a period of two years. As a result of observations carried on jointly by the Department of Rural Engineering and the Department of Poultry Husbandry of the New York State College of Agriculture, it was found that the illumination necessary for active feeding should be in the neighborhood of one foot-candle. It was also discovered that besides the illumination on the feeding area, it was quite essential that there be sufficient direct light on the perches, in order that the birds would not have a tendency to roost there.

The use of a standard 40 watt, clear, Mazda B lamp, with cone-shaped reflectors, 16 in. in diameter at the base, by 4 in. high, with reflecting surface of aluminum bronze, hung six feet above the floor and spaced 10 feet apart was found to meet the requirements.

In lighting the pen, the poultryman has at his disposal three different types of light; extending the morning light, morning and evening light, or evening light alone.

So far as production goes, each method produces satisfactory results if other conditions of management are correct. Artificial morning light is used extensively, because it is the cheapest to install, and lends itself readily to inexpensive, automatic control by a time clock. However, the extra cost of installation for evening light is small, and with this installation any method of handling may be practised. Whatever the method, the bird should have a 12 or 13 hour day.

It has been determined that the lights may be turned on suddenly without affecting the birds, but that turning the lights off suddenly makes it very difficult for the birds to go to roost.

In order to bring about a practical way for dimming the lights, the poultryman has at his disposal three different wiring systems, which are commonly known as:

1. The resistance unit system.

2. The two circuit system.

3. The series parallel system.

A full explanation of each system being given in the test.

In the concluding pages of the bulletin, data is given as to where control devices may be obtained, and the matter of the operation of poultry lighting with reference to the farm lighting plant is taken up. The bulletin is profusely illustrated with cuts which bear out the statements contained in the text, -and really tends to prove beyond a doubt the outstanding features of the bulletin. Wiring diagrams for all of the above mentioned circuits are also shown in a very simple and practical manner.-Cornell Extension Bulletin No. 40. 